This document is published in:

Composite Structures, Volume 111, May 2014, Pages 459-467.

http://dx.doi.org/10.1016/j.compstruct.2014.01.028

(C) 2014 Elsevier B.V. 


\title{
Analytical study of the low-velocity impact response of composite sandwich beams
}

\author{
Inés Ivañez ${ }^{1, *}$, Enrique Barbero ${ }^{1}$, Sonia Sanchez-Saez ${ }^{1}$ \\ Department of Continuum Mechanics and Structural Analysis, University Carlos III of Madrid, Avda. Universidad, 30, 28911 Leganes, Spain
}

\section{A R T I C L E I N F O}

\section{Article history:}

Available online $\mathrm{xxxx}$

\section{Keywords:}

Beams

Sandwich structures

Analytical modelling

Impact dynamics

Dimensional analysis

\section{A B S T R A C T}

In this work the low-velocity impact response of composite sandwich beams was studied by an analytical model. A dimensional analysis was carried out in order to identify the key parameters that influence the dynamic beam response, and to assess the effect of the dimensionless groups on the contact force and contact time. Low-velocity impact tests were conducted to validate the theoretical model. The predicted results were in good agreement with experimental data in terms of maximum contact force, contact time, and contact force-time curves. It was shown that the groups with more influence on maximum contact force and contact time are the dimensionless global stiffness, the dimensionless local stiffness, and the dimensionless impact velocity.

(ㄷ) 2014 Published by Elsevier Ltd.

\section{Introduction}

Composite sandwich structures are popular as primary structures in high-performance applications where minimum weight is essential and have widespread use in aerospace, automotive, and civil engineering industries. A reliable structural design must take into account the loads which can occur during its service life, and one area of concern is related to low-velocity impacts (i.e. tools falling during manufacturing and/or maintenance operations). Low-velocity impacts are considered potentially dangerous for a composite sandwich structure as the resulting damage is difficult to determine, especially when the face-sheets are made of carbon-fibre reinforced composite. Non or barely visible damage of a composite sandwich structure may be accompanied by substantial reduction of residual strength and stiffness [1,2]; therefore, it is needed to understand the effect of such impacts on their structural performance.

Three main approaches are used to analyse the impact response of composite sandwich structures: experimental testing, numerical simulations, and analytical models. Experimental studies have been conducted to describe the dynamic response of composite sandwich structures and investigate the impact damage produced by low-velocity impacts [3-5]; nevertheless, the amount of information obtained from experimental testing is limited and a broad

\footnotetext{
* Corresponding author. Tel.: +34 916249162; fax: +34 916248331.

E-mail address: idel@ing.uc3m.es (I. Ivañez).

1 Mechanics of Advanced Materials Research Group, web page: http:// www.uc3m.es/mma.
}

testing programme has to be undertaken to set up an accurate experimental response. Detailed finite-element models have proved to consistently predict the impact response of sandwich structures [6-9]; however, complex numerical simulations require more computational and modelling effort. A first stage to understand the effect of impacts on structures is to build a basic model for predicting the contact force history and the overall response of the impacted structure [10]. On this point, analytical simplified models lead to more efficient tools, as they can assess global variables rapidly.

Many simplified models proposed in the literature consider the balance of energy of the system [11-13]; however, the energy-balance models simplify the dynamic of structures by assuming a quasi-static behaviour at its maximum deflection. Simplified mass-springs models take into account the dynamic of the structure and present some advantages, as they rely on measurable global variables and their predictions are easier to validate.

While there are several mass-springs models for representing the response of sandwich plates subjected to low-velocity impact by hemispherical impactors [14-17], less attention has been paid to cylindrical impactors and composite sandwich beams [18]. However, many structures can be modelled as beams (i.e. windmills blades). In addition, although mass-springs models are applied to reproduce the dynamics of sandwich structures, no systematic study on the influence of the parameters that control the low-velocity process has been found. One possible approach to this kind of study is to express the equations of the model in a non-dimensional form [19]. 


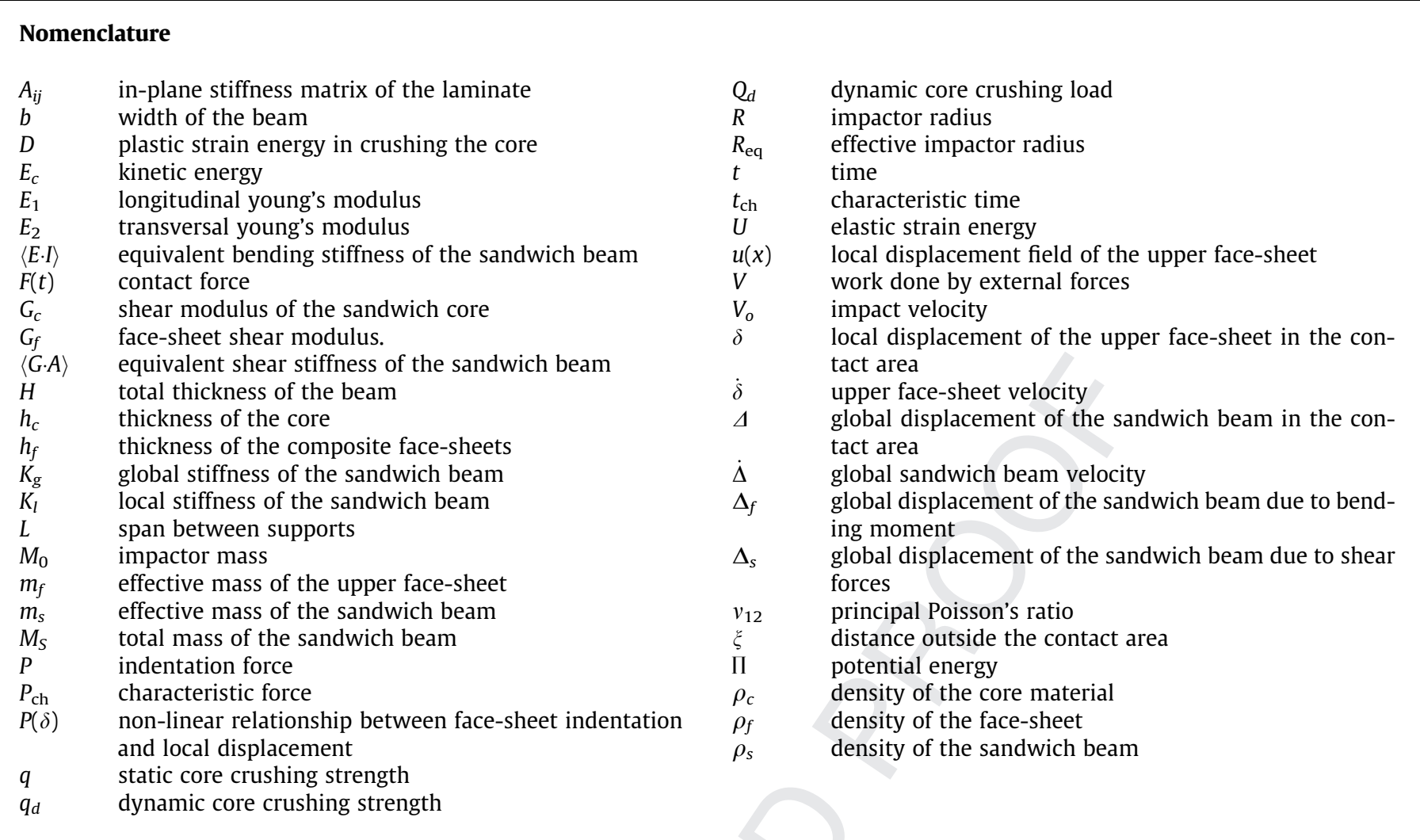

The aim of this work is to develop a non-dimensional analytical model to predict the dynamic response of composite sandwich beams subjected to low-velocity impact, prior to visible failure of the upper face-sheet. Experimental three-point bending tests were conducted to validate the model predictions. A dimensional analysis was carried out to group the key parameters of the model as dimensionless groups, and study their influence on the contact force and contact time.

\section{Model formulation}

The model developed in this work is based on Hoo Fatt and Park model [15]. The proposed model allows the analysis of the impact response of composite sandwich beams subjected to low-velocity impact, considering the effect of the nonlinear relationship between the indentation force and the local displacement of the upper face-sheet in the formulation. The effects of the inertial masses of both the upper face-sheet and the whole sandwich structure are included. Low-velocity impact tests on simplysupported sandwich beams using a cylindrical impactor (Fig. 1a) were conducted to validate the predicted results. The model is formulated in terms of dimensionless parameters in order to determine the key dimensionless groups which control the dynamic response of the sandwich beams. The formulation of the model leads to a system of nonlinear differential equations which cannot be solved analytically, and therefore requires the use of numerical methods.

The problem is modelled as a discrete system of two-degreesof-freedom (Fig. 1b). In Fig. 1b, the global stiffness of the beam is represented as a linear spring $K_{g}$ whereas to represent the local contact between the upper face-sheet and the impactor, a nonlinear local spring is employed $K_{1} . M_{0}$ is the mass of the impactor which contacts the upper face-sheet of the sandwich structure. The inertia of the upper face-sheet is represented by an effective mass $m_{f}$, and the inertia of the mass of the sandwich beam is represented by and effective mass, $m_{s}$. The core crushing load is represented by $Q_{d}$. The local displacement of the upper face-sheet and the global displacement of the whole sandwich structure are represented by $\delta$ and $\Delta$, respectively.

The equations of motion for the two-degrees-of-freedom system in Fig. $1 \mathrm{~b}$ are defined as:

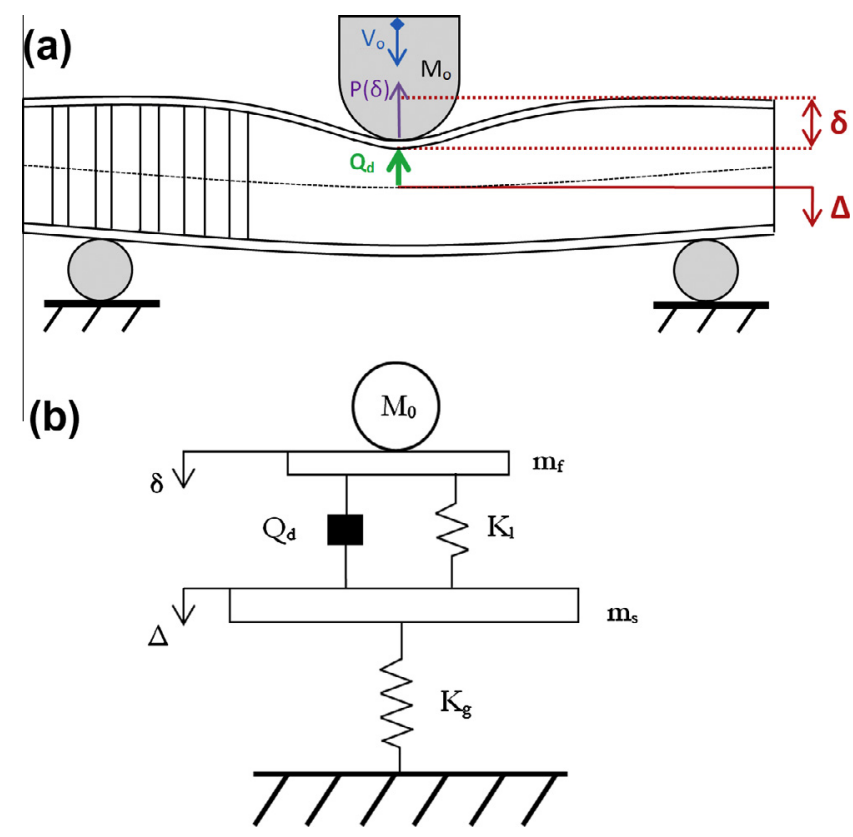

Fig. 1. Low-velocity impact on a simply-supported sandwich beam: (a) schematic representation of the problem, (b) two-degree-of-freedom mass-spring model. 


$$
\begin{aligned}
& \left(M_{0}+m_{f}\right) \cdot(\ddot{\Delta}+\ddot{\delta})+P(\delta)+Q_{d}=0 \\
& Q_{d}+P(\delta)=m_{s} \cdot \ddot{\Delta}+K_{\mathrm{gd}} \cdot \Delta
\end{aligned}
$$

To take into account the effect of the nonlinear contact between the upper face-sheet and the impactor $M_{0}$ as shown in Eq. (1), the local stiffness $K_{1}$ has been modelled as a nonlinear relationship between the indentation force and the local displacement of the upper facesheet $P(\delta)$. The system of equations shown in Eq. (1) can be solved by using the following initial conditions:

$\Delta(0)=0, \delta(0)=0, \dot{\Delta}(0)=V_{0}, \dot{\delta}(0)=0$

The only non-zero initial condition is represented by the value of the velocity of the impactor as it impacts on the sandwich structure. After solving the system of equations, the global displacement of the beam $\Delta$ and the indentation $\delta$ can be estimated. The contact force $F(t)$ is given by Eq. (3):

$F(t)=-M_{0} \cdot(\ddot{\Delta}+\ddot{\delta})$

\section{Calculation of model parameters}

The analytical model is employed to analyse the low-velocity impact response of simply-supported composite sandwich beams, although it can be used to study any sandwich structure and boundary condition by performing some minor changes in the model parameters. The methodology for determining each model parameter is briefly described as follows.

\subsection{Global stiffness of a simply-supported sandwich beam}

In a simply-supported beam subjected to a load on its central cross-section, only bending moment and shear forces appear, so the global displacement of the structure has two components due to both internal forces.

It was observed in the experimental tests that the transverse deflections of the beam are small enough so that the effects of the membrane stiffness are negligible, thus the relationship between the contact load and the global stiffness can be considered as linear. To find the global displacement of the beam, the classical equations of the Strength of Materials can be used:

$\Delta=\Delta_{f}+\Delta_{C}=\frac{F \cdot L^{3}}{48 \cdot\langle E \cdot I\rangle}+\frac{F \cdot L}{4 \cdot\langle G \cdot A\rangle}$

$\langle E \cdot I\rangle$ and $\langle G \cdot A\rangle$ are calculated using the theory of sandwich beams [20]. Therefore Eq. (4) gives the global stiffness, $K_{g}$ :

$K_{g}=\frac{48 \cdot\langle E \cdot I\rangle \cdot\langle G \cdot A\rangle}{\langle G \cdot A\rangle \cdot L^{3}+12 \cdot\langle E \cdot I\rangle \cdot L}$

3.2. Non-linear relationship between the indentation force and the local displacement of the upper face-sheet

The local indentation due to impact was modelled as the indentation produced by an impactor on a membrane which rests on a rigid-plastic foundation (Fig. 2). The membrane and the foundation represent the upper face-sheet and honeycomb core, respectively.

The relationship between the contact force and the local displacement of the beam can be found by minimising the total potential energy of the sandwich beam. The potential energy is given by:

$\Pi=U+D-V$

Prior to the calculation of the energies involved in Eq. (6), it is necessary to define a function which represents the displacement of the upper face-sheet during the impact, $u(x)$. The impactor nose
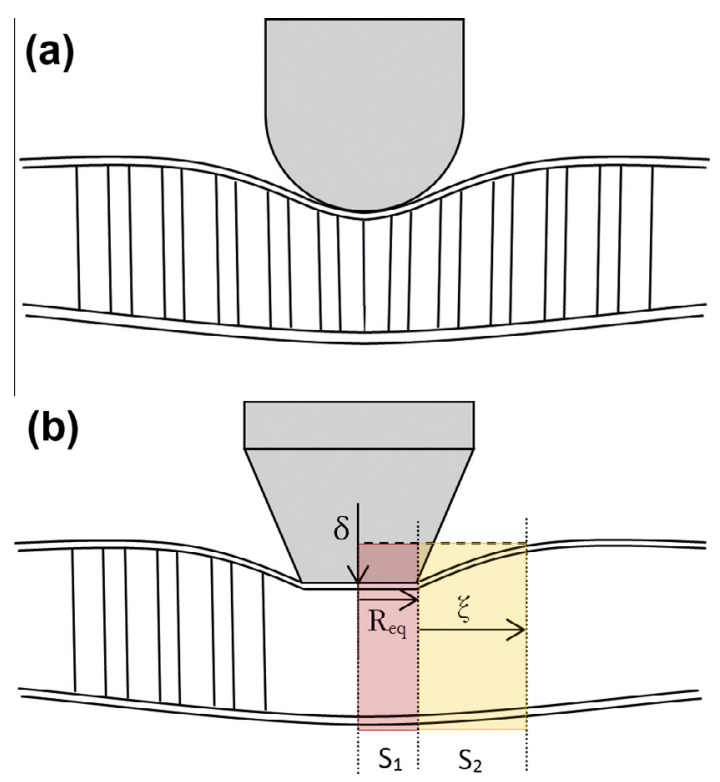

Fig. 2. Contact between the upper face-sheet and the impactor: (a) real contact, (b) assumed approximation.

had a cylindrical shape, thus the contact area can be represented by Fig. 2a. Nevertheless, an approximate shape of the real contact area is proposed in this model (Fig. 2b). The displacement field can be described by a quadratic function:

$u(x)\left\{\begin{array}{l}\delta \text { para } 0<x<R_{\text {eq }} \\ \delta \cdot\left[1-\frac{\left(x-R_{\mathrm{eq}}\right)}{\left(\xi-R_{\mathrm{eq}}\right)}\right]^{2} \text { para } R_{\mathrm{eq}}<x<\xi\end{array}\right.$

Eq. (7) is expressed in terms of the local displacement $\delta$ and the radial distance outside the contact $\xi$ (Fig. 2b). Assuming the displacement field shown in Eq. (7), the elastic strain energy of the membrane is given by:

$\begin{aligned} U= & \frac{1}{8} \iint\left[A_{11} \cdot\left(\frac{\partial u(x)}{\partial x}\right)^{4}+A_{22} \cdot\left(\frac{\partial u(x)}{\partial y}\right)^{4}\right. \\ & \left.+\left(2 \cdot A_{12}+4 \cdot A_{66}\right) \cdot\left(\frac{\partial u(x)}{\partial x}\right)^{2}\left(\frac{\partial u(x)}{\partial y}\right)^{2}\right] \mathrm{ds}\end{aligned}$

the work due to core crushing can be approximated to:

$D=\iint q_{d} \cdot u(x) \cdot \mathrm{ds}$

and the work given by the external forces to:

$V=\iint \frac{P}{2 \cdot b \cdot \rho} \cdot u(x) \cdot \mathrm{ds}$

In order to minimise the potential energy in Eq. (6), the integrals presented in Eqs. (8)-(10) must be determined and computed on two regions of the sandwich beam (Fig. 2b): the area which corresponds to the contact area between the impactor and the face-sheet $\left(S_{1}\right)$, and the area which represents the region of the upper facesheet which is not in contact with the impactor and moves due to the impact event $\left(S_{2}\right)$. Both regions are assumed to have the shape of a rectangle:

$\int_{S 1} \mathrm{ds}=\int_{0}^{b} \int_{0}^{R_{\mathrm{eq}}} \mathrm{dr} \cdot \mathrm{dy}$

$\int_{S 2} \mathrm{ds}=4 \cdot \int_{0}^{b} \int_{R_{\mathrm{eq}}}^{\xi} \mathrm{dx} \cdot \mathrm{dy}$ 
In this case, it is considered that the width of the impactor is coincident with the width of the sandwich beam, $b$.

Substituting Eqs. (7), (11), and (12) into Eq. (8) gives the following expression for the elastic strain energy:

$U=\frac{1}{8} \int_{S 2}\left[A_{11} \cdot\left(\frac{\partial u(x)}{\partial x}\right)^{4}\right] \mathrm{ds}=\frac{8}{5} \cdot \frac{b \cdot A_{11} \cdot \delta^{4}}{\left(\xi-R_{\mathrm{eq}}\right)^{3}}$

The work due to the crushing of the core is calculated as:

$$
\begin{aligned}
D & =\int_{S 1} q_{d} \cdot u(x) \cdot \mathrm{ds}+\int_{S 2} q_{d} \cdot u(x) \cdot \mathrm{ds} \\
& =q_{d} \cdot \delta \cdot b \cdot R_{\mathrm{eq}}+q_{d} \cdot \delta \cdot b \cdot \frac{\left(\xi-R_{\mathrm{eq}}\right)}{3}
\end{aligned}
$$

To calculate the external work produced by indentation, it is assumed that it has only a local effect and; thus, there is only a component in the region of contact between the impactor and the upper face-sheet $\left(S_{1}\right)$ :

$V=\int_{S 1} \frac{P}{b \cdot 2 \cdot R_{\mathrm{eq}}} \cdot u(x) \cdot \mathrm{ds}=\frac{P}{2} \cdot \delta$

Substituting Eq. (13)-(15) into Eq. (6), the potential energy is given by:

$\Pi=\frac{8}{5} \cdot \frac{b \cdot A_{11} \cdot \delta^{4}}{\left(\xi-R_{\mathrm{eq}}\right)^{3}}+q_{d} \cdot \delta \cdot b \cdot R_{\mathrm{eq}} \cdot+q_{d} \cdot \delta \cdot b \cdot \frac{\left(\xi-R_{\mathrm{eq}}\right)}{3}-\frac{P}{2} \cdot \delta$

Eq. (16) is expressed in terms of $\delta$, the distance $\xi$, and $P$. From the minimum condition of $\Pi$ with respect to $\delta$, it can be found:

$P(\delta, \xi)=2 \cdot b \cdot\left[\frac{32}{5} \cdot \frac{A_{11} \cdot \delta^{3}}{\left(\xi-R_{\mathrm{eq}}\right)^{3}}+q_{d} \cdot 2 \cdot R_{\mathrm{eq}}+q_{d} \cdot \frac{\left(\xi-R_{\mathrm{eq}}\right)}{3}\right]$

Minimising Eq. (17) with respect to $\xi$, and eliminating $\xi$ from the result, gives the nonlinear relationship between the indentation force and the local displacement:

$P(\delta)=2 \cdot b \cdot q_{d}\left[R_{\mathrm{eq}}+\frac{4}{9}\left[\frac{288 \cdot A_{11} \cdot \delta^{3}}{5 \cdot q_{d}}\right]^{1 / 4}\right]$

This expression can be expressed as:

$P(\delta)=\alpha+\beta \cdot \delta^{3 / 4}$

where

$\alpha=2 \cdot b \cdot q_{d} \cdot R_{\mathrm{eq}}$.

$\beta=\frac{8}{9} \cdot b \cdot\left[\frac{288 \cdot q_{d}^{3} \cdot A_{11}}{5}\right]^{1 / 4}$

\subsection{Dynamic core crushing load}

The dynamic crushing load of the core depends on the contact between the impactor and the core. Using the hypothesis showed in Fig. $2 b, Q_{d}$ can be estimated by:

$Q_{d}=2 \cdot R_{\mathrm{eq}} \cdot b \cdot q_{d}$

\subsection{Effective masses}

The effective mass of the upper face-sheet can be calculated by assuming that the velocity profile is similar to the face-sheet displacement field [15]. If the composite face-sheets are in membrane state, the velocity profile is given by the derivative of Eq. (7) with respect to time. Thus, the kinetic energy can be expressed approximately as:
$E_{C} \approx b \cdot \rho_{f} \cdot h_{f} \cdot \dot{\delta}^{2} \cdot\left[R_{\mathrm{eq}}+8 \cdot \frac{\left(\xi-R_{\mathrm{eq}}\right)}{5}\right]$

The kinetic energy of the effective mass of the upper face-sheet can be expressed as follows:

$E_{C}=\frac{1}{2} \cdot m_{f} \cdot \dot{\delta}^{2}$

Therefore the effective mass of the upper face-sheet is:

$m_{f}=2 \cdot b \cdot \rho_{f} \cdot h_{f} \cdot\left[R_{\mathrm{eq}}+8 \cdot \frac{\left(\xi-R_{\mathrm{eq}}\right)}{5}\right]$

Assuming that the low-velocity impact deflection affects to a $25 \%$ of the sandwich beam [21], $\xi$ is supposed to be equal to $L / 4$ :

$m_{f}=2 \cdot \rho_{f} \cdot h_{f} \cdot b \cdot\left[R_{\mathrm{eq}}+8 \cdot \frac{\left(\frac{L}{4}-R_{\mathrm{eq}}\right)}{5}\right]$

Similarly, to calculate the effective mass of the sandwich beam, the velocity profile can be approximated to the displacement profile of a beam loaded at its centre [22]. The kinetic energy can be approximated to:

$E_{C} \approx \frac{2}{3} \cdot b \cdot \rho_{s} \cdot\left(h_{c}+2 \cdot h_{f}\right) \cdot L \cdot \dot{\Delta}^{2}$

The kinetic energy of the effective mass of the sandwich beam is given by:

$E_{C}=\frac{1}{2} \cdot m_{s} \cdot \dot{\Delta}^{2}$

The effective mass of the sandwich beam is obtained by combining Eq. (27) with Eq. (28):

$m_{s}=\frac{4}{3} \cdot b \cdot \rho_{s} \cdot\left(h_{c}+2 \cdot h_{f}\right) \cdot L$

\section{Dimensionless formulation of the model}

The system of differential equations showed in Eq. (1) represents the motion of the sandwich beam, and several parameters appear in this equation. To determine which parameters are most relevant in the low-velocity impact response, the model is formulated in terms of dimensionless variables and groups.

\subsection{Definition of the dimensionless groups}

The dimensionally independent units used in this analysis are defined as: the impactor mass $\left(M_{0}\right)$, the thickness of the sandwich beam $(H)$, and the thickness of the upper face-sheet $\left(h_{f}\right)$. The time variable and the contact force are dimensionless through use a characteristic time and a characteristic force, respectively. The resulting dimensionless variables are shown in Table 1.

The characteristic time $\left(t_{\mathrm{ch}}\right)$ is defined as the inverse of the frequency corresponding to the first mode of vibration of a simply-supported beam:

$t_{\mathrm{ch}}=\frac{\sqrt{M_{S} \cdot L^{3}}}{\pi^{2} \cdot \sqrt{\langle E \cdot I\rangle}}$

In case of slender beams, the effect of the equivalent shear stiffness in Eq. (30) is neglected.

Table 1

Dimensionless variables.

\begin{tabular}{cccc}
\hline$\hat{\delta}$ & $\widehat{\Delta}$ & $\widehat{F}$ & $\hat{t}$ \\
\hline$\frac{\delta}{h_{f}}$ & $\frac{\Delta}{H}$ & $\frac{F}{P_{c h}}$ & $\frac{t}{t_{c h}}$ \\
\hline
\end{tabular}


The dimensionless groups used in the model analysis are obtained when the equations of motion (Eq. (1)) are formulated in terms of dimensionless variables, and are presented in Table 2.

The physical meaning of each dimensionless group is explained as follows:

- $\Pi_{1}$ : Represents the relationship between the effective mass of the upper face-sheet and the mass of the impactor.

- $\Pi_{2}$ : The dimensionless thickness $\hat{h}$, relates the thickness of the upper face-sheet with the thickness of the sandwich beam.

$-\Pi_{3}$ : Represents the relationship between the effective mass of the sandwich beam and the mass of the impactor.

- $\Pi_{4}$ : The dimensionless global stiffness $\widehat{K_{g}}$ relates the equivalent bending stiffness with the equivalent shear stiffness of the sandwich beam.

- $\Pi_{5}$ : Represents the relationship between the dynamic core crushing load $Q_{d}$, and the equivalent bending stiffness of the sandwich beam.

- $\Pi_{6}$ : The dimensionless parameter $\beta(\hat{\beta})$ relates the nonlinear relationship between the indentation force and the local displacement with the equivalent bending stiffness of the beam. $P(\delta)$ depends on two parameters $\alpha$ and $\beta$, thus it is needed to convert to dimensionless form both terms. However, dimensionless $\alpha(\hat{\alpha})$ shows the same formulation as the previously defined $\Pi_{5}$, and therefore the effect of $\hat{\alpha}$ on the analysis is only taken into account in Eq. (31).

- $\Pi_{7}$ : The dimensionless initial velocity relates the initial velocity of the impactor with a reference velocity, which is defined by the first mode of vibration of a simply-supported beam, and the sandwich thickness.

Using the dimensionless groups, the non-dimensional equations of motion are given by:

$$
\begin{gathered}
\left(1+\Pi_{1}\right) \cdot\left(\ddot{\widehat{\Delta}}+\Pi_{2} \cdot \ddot{\hat{\delta}}\right)+\Pi_{6} \cdot \widehat{\delta^{3 / 4}}+2 \cdot \Pi_{5}=0 \\
\Pi_{6} \cdot \widehat{\delta^{3 / 4}}+2 \cdot \Pi_{5}=\Pi_{3} \cdot \ddot{\widehat{\Delta}}+\Pi_{4} \cdot \widehat{\Delta}
\end{gathered}
$$

When $P_{\text {ch }}$ is defined as:

$P_{\mathrm{ch}}=\frac{M_{0}}{M_{s}} \cdot \frac{H}{4 \cdot L^{3}} \cdot\langle E \cdot I\rangle$

The initial conditions, show in Eq. (2), are also converted into a dimensionless form by using $\Pi_{7}$ :

$\widehat{\Delta}(0)=0, \dot{\widehat{\Delta}}(0)=\frac{V_{0} \cdot t_{\mathrm{ch}}}{H}=\Pi_{7}, \hat{\delta}(0)=0, \dot{\hat{\delta}}(0)=0$

The system of equations presented in Eq. (31) is nonlinear and it cannot be solved analytically, thus it is needed to use numerical methods. In this work the Runge-Kutta method is used to solve the equations of motion.

\section{Model validation}

In order to validate the analytical model, dynamic three-point bending tests were carried out in an instrumented drop-weight tower, from which the contact force during the impact event was recorded.

Table 2

Dimensionless groups.

\begin{tabular}{ccccccc}
\hline$\hat{m}_{f}$ & $\hat{h}$ & $\widehat{m}_{s}$ & $\widehat{K}_{g}$ & $\widehat{Q_{d}}$ & $\hat{\beta}$ & $\widehat{V}_{0}$ \\
\hline$m_{f}$ & $\frac{h_{f}}{H}$ & $\frac{m_{s}}{M_{0}}$ & $\frac{K_{g} \cdot H}{P_{\mathrm{ch}}}$ & $\frac{Q_{d}}{P_{\mathrm{ch}}}$ & $\frac{\beta \cdot h_{f}^{3 / 4}}{P_{\mathrm{ch}}}$ & $\frac{V_{0} \cdot t_{\mathrm{ch}}}{H}$ \\
$\Pi_{1}$ & $\Pi_{2}$ & $\Pi_{3}$ & $\Pi_{4}$ & $\Pi_{5}$ & $\Pi_{6}$ & $\Pi_{7}$ \\
\hline
\end{tabular}

\subsection{Experimental procedure}

Composite sandwich beams of rectangular cross-section ( $50 \mathrm{~mm}$ in width and $24 \mathrm{~mm}$ in thickness) were tested at different impact velocities using a span of $430 \mathrm{~mm}$. The impactor mass and the nose radius were $3.966 \mathrm{~kg}$ and $20 \mathrm{~mm}$, respectively. The low-velocity impact tests were recorded by a high-speed camera.

The sandwich face-sheets were made of plain woven laminate of carbon fibre and epoxy resin (AS4-8552). The thickness of the each face-sheet was $2 \mathrm{~mm}$. The core consisted of 3003 alloy hexagonal aluminium honeycomb, with a thickness and a cell-size of $20 \mathrm{~mm}$ and $4.8 \mathrm{~mm}$ respectively.

The main characteristics of both the face-sheet and the core materials are shown in Table 3.

\subsection{Validation results}

The model validation was carried out by comparing the analytical results with the experimental data in terms of contact forcetime curves, maximum contact force, and contact time. The comparison was performed for impact velocities for which no visible failure of the composite upper face-sheet occurs (between $2.04 \mathrm{~m} / \mathrm{s}$ and $3.00 \mathrm{~m} / \mathrm{s}$ ). The initial velocity of the impactor was measured by using the high-speed camera recordings.

Analytical and experimental force-time curves obtained for three different impact velocities are presented in Fig. 3. The analytical model curves show the presence of two natural modes, each with a separate resonance frequency. These oscillations have already been observed in two-degrees-of-freedom models by other researchers [11].

The analytical and experimental contact force-time curves show a similar trend in terms of maximum peak force, and contact time. The comparison between the predicted maximum contact force and the experimental measurements is presented in Table 4.

The difference between the experimental and analytical results is less than $8 \%$, thus the analytical maximum contact force results are within a reasonable range of prediction. The comparison between the predicted and measured contact time values is shown in Table 5, being the difference less than $6 \%$ in the three cases.

The analytical results are in good agreement with the experimental data, thus the analytical model can reproduce the dynamic bending response of composite sandwich beams subjected to lowvelocity impact when the damage on the upper face-sheet is not extensive, and therefore more difficult to detect.

\section{Discussion and results}

After the validation of the analytical model, a study of the influence of the dimensionless groups on the maximum contact force and contact time was carried out. The dimensional analysis is performed by varying every dimensionless group presented in Table 2 .

Table 3

Material properties of the lamina and the honeycomb core.

\begin{tabular}{ll}
\hline Lamina & \\
$E_{1}$ & $68.9 \mathrm{GPa}$ \\
$E_{2}$ & $68.9 \mathrm{GPa}$ \\
$G_{f}$ & $9 \mathrm{GPa}$ \\
$v_{12}$ & 0.22 \\
$\rho_{f}$ & $1600 \mathrm{~kg} / \mathrm{m}^{3}$ \\
Core & \\
$\rho_{c}$ & $77 \mathrm{~kg} / \mathrm{m}^{3}$ \\
$h_{c}$ & $20 \mathrm{~mm}$ \\
$G_{c}$ & $144 \mathrm{MPa}$ \\
\hline
\end{tabular}



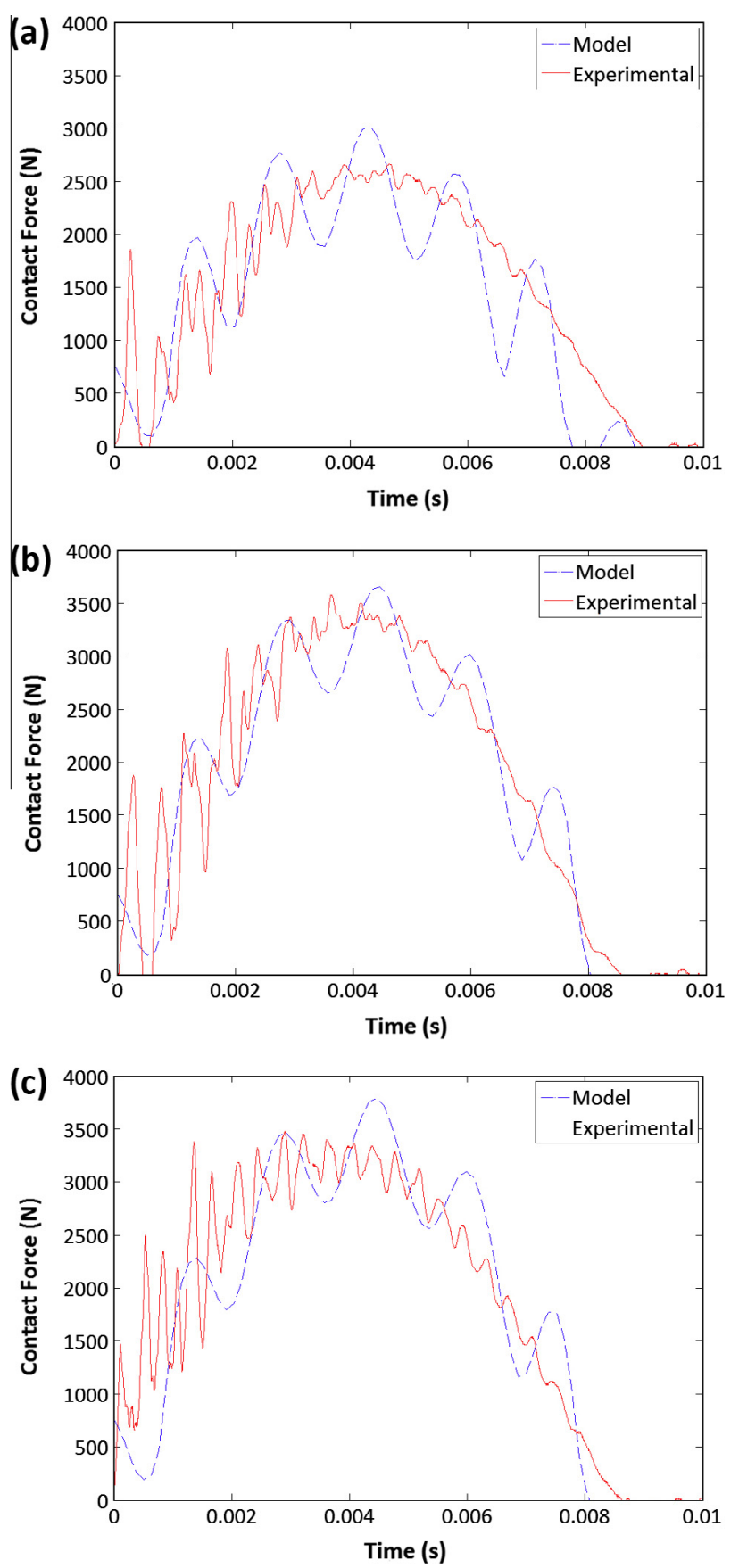

Fig. 3. Comparison between the experimental and predicted contact force versus time curves. Impact velocity: (a) $2.04 \mathrm{~m} / \mathrm{s}$, (b) $2.62 \mathrm{~m} / \mathrm{s}$, and (c) $2.77 \mathrm{~m} / \mathrm{s}$.

Table 4

Experimental and predicted maximum contact force results.

\begin{tabular}{llll}
\hline \multirow{2}{*}{ Impact velocity $(\mathrm{m} / \mathrm{s})$} & \multicolumn{2}{l}{ Maximum contact force $(\mathrm{N})$} & \multirow{2}{*}{ Difference $(\%)$} \\
\cline { 2 - 3 } & Experimental & Prediction & \\
\hline 2.04 & 2671.20 & 2578.70 & 3.46 \\
2.62 & 3587.04 & 3413.10 & 7.81 \\
2.77 & 3482.10 & 3597.80 & 3.32 \\
\hline
\end{tabular}

\subsection{Dimensional analysis of the model}

The limits of the studied variation range for the dimensionless groups are between an order of magnitude above and below the validation experimental value (reference value). Therefore, the
Table 5

Experimental and predicted contact time results.

\begin{tabular}{llll}
\hline \multirow{2}{*}{ Impact velocity $(\mathrm{m} / \mathrm{s})$} & \multicolumn{2}{l}{ Contact time $(\mathrm{ms})$} & Difference $(\%)$ \\
\cline { 2 - 3 } & Experimental & Prediction & \\
\hline 2.04 & 8.96 & 8.78 & 2.00 \\
2.62 & 8.56 & 8.11 & 5.25 \\
2.77 & 8.58 & 8.21 & 4.31 \\
\hline
\end{tabular}

effects of such variations on the low-velocity impact response of the sandwich beams can be computed for a broad range. However, it was not possible to complete the selected range for $\mathrm{I}_{2}$, as convergence problems occur at some point during the calculations.

The percentage of the variation in the maximum contact force and the contact time that results from the variation of each dimensionless group between the upper and lower limit of the studied range, is shown in Table 6.

The dimensionless groups with more influence on the variation of the maximum contact force and the contact time within the studied range are $\Pi_{4}, \Pi_{6}$ and $\Pi_{7}$. These groups correspond to the dimensionless global stiffness, the dimensionless parameter $\beta$ and the dimensionless impact velocity, respectively.

The dimensionless effective masses corresponding to the upper face-sheet $\left(\Pi_{1}\right)$ and the sandwich beam $\left(\Pi_{2}\right)$ showed less variation in comparison with the previous groups, although their influence on the dynamics of the system should not be considered negligible (variations observed in the studied range are above 10\%).

The following sections show in more detail the results for the three dimensionless groups with more influence on the contact force-time curves.

\subsection{Analysis of the dimensionless global stiffness}

As previously stated, the dimensionless group $\Pi_{4}$ represents the relationship between the equivalent bending stiffness and he equivalent shear stiffness of the beam. The variation of the dimensionless global stiffness of the sandwich beam, strongly affects to the results both in terms of maximum contact force, and contact time.

Increasing $\Pi_{4}$ increases the maximum contact force, and decreases the contact time when assessing the low-velocity impact response of composite sandwich beams. The variation percentage between the lower and upper limit in terms of maximum contact force is $81.21 \%$. It was observed that the maximum contact force increases more rapidly until $\Pi_{4}$ is close to unity. From this value, the slope of the curve diminishes, and changes are less noticeable (Fig. 4).

The contact time- $\Pi_{4}$ curve (Fig. 5) displays a different trend. The lower limit of the selected range $\left(6.35 \cdot 10^{-2}\right)$ shows the largest contact time of all the cases studied $(30 \mathrm{~ms})$. At the beginning of the curve, the contact time decreased rapidly; however, when $\Pi_{4}$ reaches a value close to unity, the contact time diminishes less rapidly. Therefore, $\Pi_{4}$ is not significant in both maximum contact force and contact time for values greater than approximately 1 .

In the analysed range, the maximum contact force can be fitted to a potential function with a correlation coefficient of 0.97 , whereas the contact time could be fitted using a logarithmic function with a correlation coefficient of 0.94 .

\subsection{Analysis of the dimensionless local stiffness}

The dimensionless group $\Pi_{6}$ relates the nonlinear relationship between the indentation force and the local displacement, $P(\delta)$, with the equivalent bending stiffness of the sandwich beam. $P(\delta)$ is related to the local stiffness of the sandwich beam during the impact.

An increase of $\Pi_{6}$ increases the maximum contact force, and decreases the contact time. Variations in both results are observed 
Table 6

Percentage of variation in maximum contact force and contact time for the studied dimensionless groups.

\begin{tabular}{|c|c|c|c|c|c|}
\hline Dimensionless group & Lower limit & Reference value & Upper limit & Variation on the maximum contact force (\%) & Variation on the contact time (\%) \\
\hline$\Pi_{1}$ & $3.25 \cdot 10^{-3}$ & $3.25 \cdot 10^{-2}$ & $3.25 \cdot 10^{-1}$ & 14.41 & 16.91 \\
\hline$\Pi_{2}$ & $7.66 \cdot 10^{-2}$ & $8.33 \cdot 10^{-2}$ & $1.00 \cdot 10^{-1}$ & 3.81 & 3.92 \\
\hline$\Pi_{3}$ & $5.74 \cdot 10^{-3}$ & $5.74 \cdot 10^{-2}$ & $5.74 \cdot 10^{-1}$ & 10.25 & 10.67 \\
\hline$\Pi_{4}$ & $6.35 \cdot 10^{-2}$ & $6.35 \cdot 10^{-1}$ & $6.35 \cdot 10^{0}$ & 81.21 & 85.43 \\
\hline$\Pi_{5}$ & $2.19 \cdot 10^{-3}$ & $2.19 \cdot 10^{-2}$ & $1.96 \cdot 10^{-1}$ & 5.19 & 1.21 \\
\hline$\Pi_{6}$ & $2.21 \cdot 10^{-2}$ & $2.21 \cdot 10^{-1}$ & $2.21 \cdot 10^{0}$ & 94.59 & 50.00 \\
\hline$\Pi_{7}$ & $9.92 \cdot 10^{-2}$ & $2.66 \cdot 10^{-1}$ & $3.00 \cdot 10^{-1}$ & 202.78 & 2.04 \\
\hline
\end{tabular}

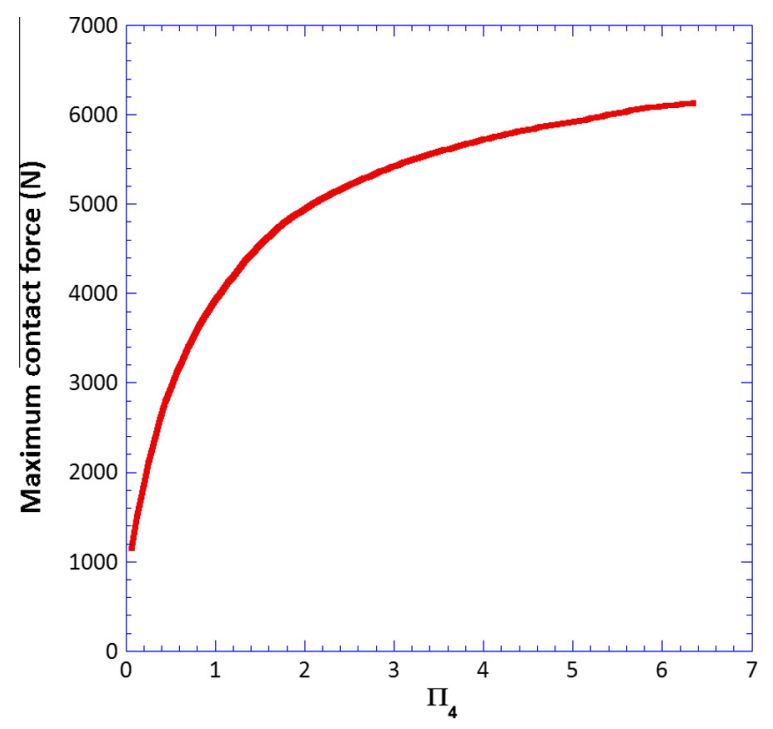

Fig. 4. Maximum contact force versus variation of the dimensionless group $\Pi_{4}$.

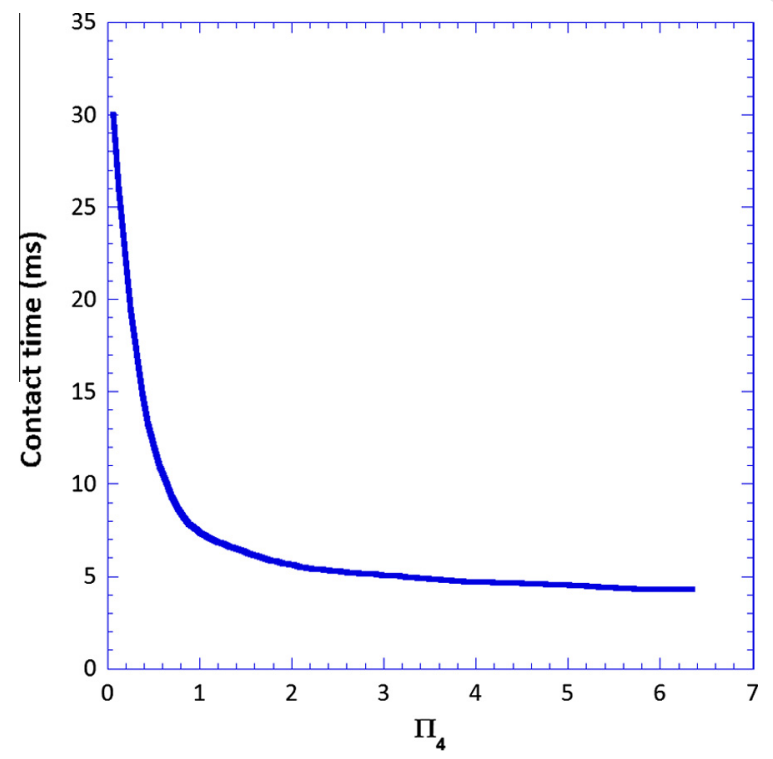

Fig. 5. Contact time versus variation of the dimensionless group $\Pi_{4}$.

until the reference value is approximately reached $\left(2.21 \cdot 10^{-2}\right)$, as shown in Figs. 6 and 7. From this point, $\Pi_{6}$ influence on the results is less important. The variation percentage is higher for the maximum contact force $(94.59 \%)$ than for the contact time (50\%).

At the studied range, the contact time could be fitted to a potential function with a correlation coefficient of 0.93 and the maximum contact force can be fitted to a logarithmic function with a correlation coefficient of 0.92 .

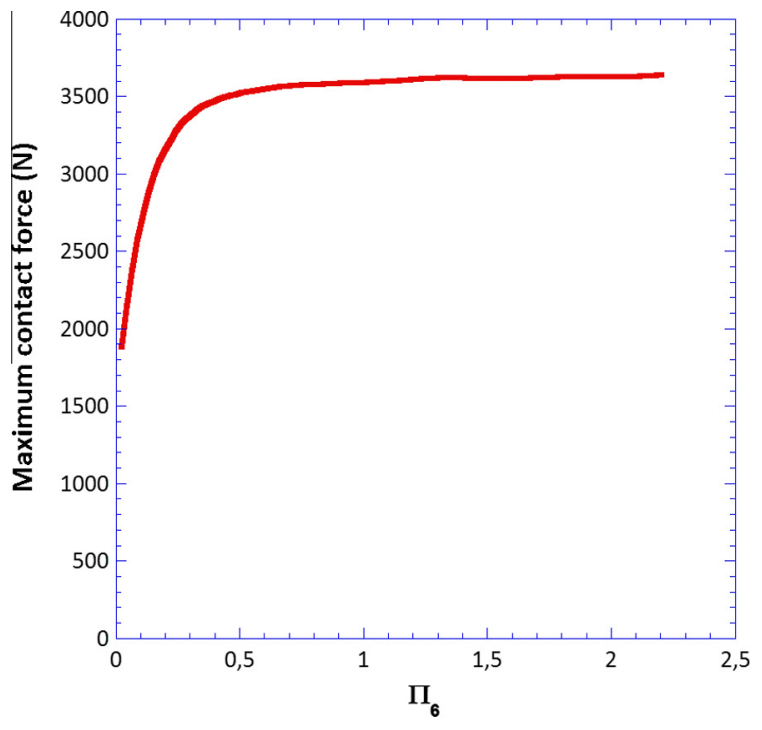

Fig. 6. Maximum contact force versus variation of the dimensionless group $\Pi_{6}$.

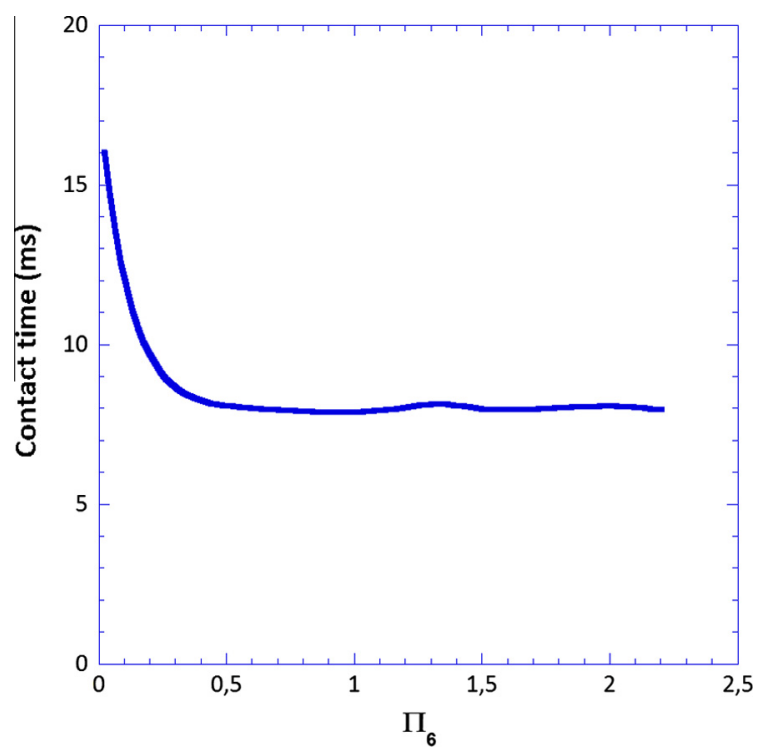

Fig. 7. Contact time versus variation of the dimensionless group $\Pi_{6}$.

\subsection{Analysis of the dimensionless initial velocity}

To represent the dimensionless initial conditions, the dimensionless group $\Pi_{7}$ was defined. The initial velocity of the impactor (impact velocity) is the only non-zero initial condition, as shown in Eq. (33).

The reference value of group $\Pi_{7}(0.26)$ is given by a velocity impact of $2.77 \mathrm{~m} / \mathrm{s}$, and corresponds to an impact energy of 
approximately $15 \mathrm{~J}$. This model is used for predicting the lowvelocity impact response of composite sandwich beams with barely visible damage after impact; thus the upper limit studied is 0.30 , which corresponds to impact energy of $20 \mathrm{~J}$. The experimental upper face-sheet failure occurred at impact energy $21 \mathrm{~J}$.

The dimensionless initial velocity strongly affects to the maximum contact force (Fig. 8), but shows little effect on the contact time (Fig. 9). The contact force increases 3 times between the upper and the lower limit of the studied $\Pi_{7}$ range, thus the variations on the maximum contact force results are noticeable in the selected range. However, the contact time remains almost contact for all the studied $\Pi_{7}$, and differences are not higher than $10 \%$ (Fig. 9).

Between the selected limits, the variation of the maximum contact force as a function of $\Pi_{7}$ gives a straight line, which depicts a linear trend in the predicted data. The results can be fitted to a linear adjustment with a correlation coefficient of 0.99 . The contact

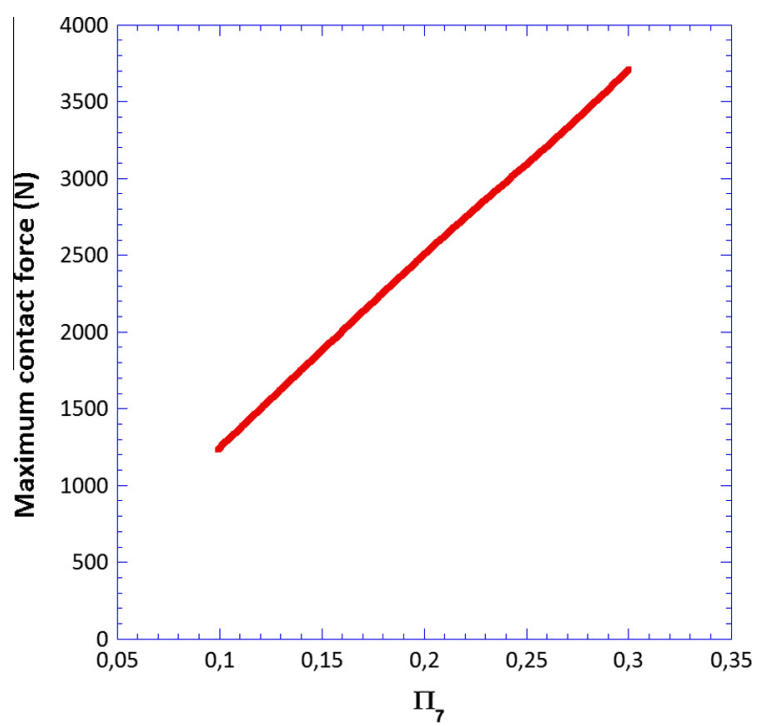

Fig. 8. Maximum contact force versus variation of the dimensionless group $\Pi_{7}$.

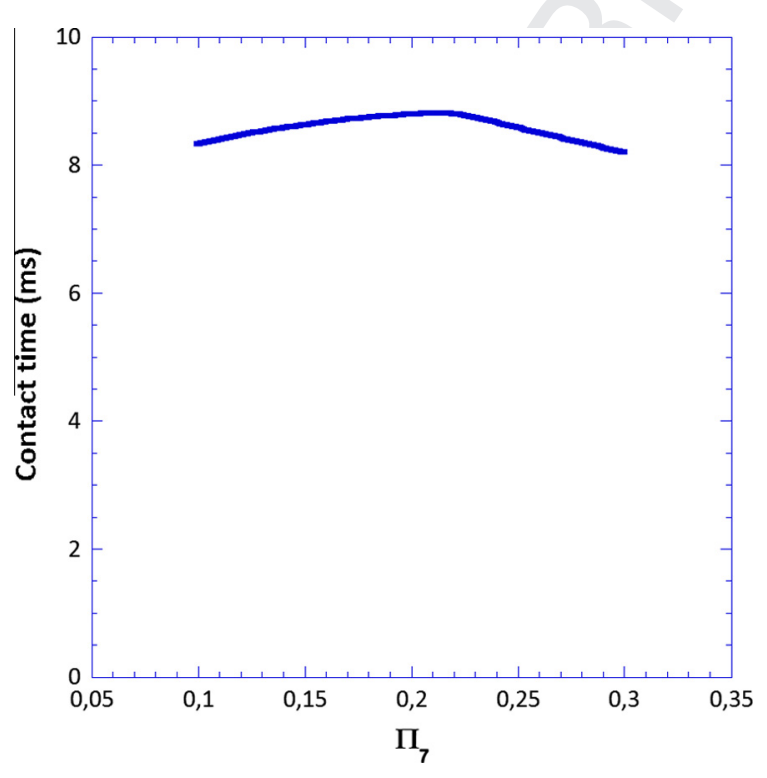

Fig. 9. Contact time versus variation of the dimensionless group $\Pi_{7}$. time reaches a maximum at approximately $\Pi_{7}=0.22$ and has no defined trend.

\section{Conclusions}

In this work the low-velocity impact response of composite sandwich beams was studied by a two-degrees-of-freedom massspring model. The dimensionless formulation of the model allowed determining the key dimensionless groups and permitted to evaluate the response of sandwich beams subjected to dynamic loads if the damaged area on the upper face-sheet is not extensive, and does not affect significantly to the global stiffness of the beam. The model predictions are in good agreement with the experimental results.

In the analysed range, the groups with more influence in terms of maximum contact force and contact time are: the dimensionless global stiffness, the dimensionless parameter $\beta$, which is part of the nonlinear relationship between the indentation force and the local displacement and the dimensionless impact velocity. Both dimensionless effective mass of the upper face-sheet and dimensionless effective mass of the whole sandwich beam have less influence on the system response, although their influence should not be considered negligible.

Decreasing the dimensionless global stiffness of the structure reduces the maximum contact force and increases the contact time; however, the results showed that $\Pi_{4}$ is not significant in the system response for values greater than approximately 1 . With the increasing dimensionless global stiffness of the structure, the response tends to stabilise both in maximum contact force and contact time values.

Dimensionless $\beta$ also has a significant influence on the system. Below approximately the reference value, $\Pi_{6}$ has a significant effect on both maximum contact force and contact time results. Increasing the dimensionless parameter, results in a rapid increase of the maximum contact force, until it stabilises around a constant value. On the contrary, the contact time shows a strong decrease until reaching a stable value.

Finally, it was observed that increasing the dimensionless initial velocity causes noticeable increase in the maximum contact force, which was adequately represented by linear regression. However, the contact time remains constant around certain values. As a result, $\Pi_{7}$ shows more influence on the maximum contact force than on the contact time results.

\section{References}

[1] Zonghong X, Vizzini AJ, Qingru T. On residual compressive strength prediction of composite sandwich panels after low-velocity impact damage. Acta Mech Sol Sinica 2006;19(1):9-17.

[2] Davies GAO, Hitchings D, Besant T, Clarke A, Morgan C. Compression after impact strength of composite sandwich panels. Compos Struct 2004;63:1-9.

[3] Mines RAW, Worrall CM, Gibson AG. Low velocity perforation behavior of polymer composite sandwich panels. Int J Impact Eng 1998;21:855-79.

[4] Anderson T, Madenci E. Experimental investigation of low-velocity impact characteristics of sandwich composites. Compos Struct 2000;50:239-47.

[5] Schubel PM, Luo J-J, Daniel IM. Low-velocity impact behaviour of composite sandwich panels. Compos Part A 2005;36(10):1389-96.

[6] Foo CC, Chai GB, Seah LK. A model to predict low-velocity impact response and damage in sandwich composites. Compos Sci Technol 2008;68:1348-56.

[7] Icardi U, Ferrero L. Impact analysis of sandwich composites based on a refined plate element with strain energy updating. Compos Struct 2009;89:35-51.

[8] Ivañez I, Santiuste C, Sanchez-Saez S. FEM analysis of dynamic flexural behaviour of composite sandwich beams with foam core. Compos Struct 2010;92:2285-91.

[9] Ivañez I, Sanchez-Saez S. Numerical modelling of the low-velocity impact response of composite sandwich beams with honeycomb core. Compos Struct 2013;106:716-23.

[10] Abrate S. Modeling of impacts on composite structures. Compos Struct 2001;51:129-38.

[11] Akil Hazizan MD, Cantwell WJ. The low velocity impact response of foambased sandwich structures. Compos Part B 2002;33:193-204. 
[12] Abrate S. Localized impact on sandwich structures with laminated facings. Appl Mech Rev 1997;50:70-82.

[13] Crupi V, Epasto G, Guglielmino E. Collapse modes in aluminium honeycomb sandwich panels under bending and impact loading. Int J Impact Eng 2012;43:6-15.

[14] Gustin J, Mahinfalah M, Jazar Nakhaie G, Aagaah MR. Low-velocity impact of sandwich composite plates. Soc Exp Mech 2004;44(6):574-83.

[15] Hoo Fatt MS, Park KS. Dynamic models for low velocity impact damage of composite sandwich panels - Part A: deformation. Compos Struct 2001;52:335-51.

[16] Nettles AT, Lance DG. Enhancement of impact damage tolerance of composite laminates. Compos Eng 1993;3:383-94.
[17] Türk MH, Hoo Fatt MS. Localized damage response of composite sandwich plates. Compos Part B 1999;30:157-65.

[18] Aprete NA, Sankar BV, Ambur DR. Low-velocity impact response of sandwich beams with functionally graded core. Int J Sol Struct 2006;43:2479-96.

[19] Garcia-Castillo SK, Sanchez-Saez S, Barbero E. Nondimensional analysis of ballistic impact on thin woven laminate plates. Int J Impact Eng 2012;39(1):8-15.

[20] Zenkert D. An Introduction to Sandwich Construction. UK: EMAS Ltd.; 1995.

[21] Iváñez I. Análisis y modelización de vigas sándwich sometidas a impactos de baja velocidad. PhD Thesis, University Carlos III Madrid; July 2013.

[22] Dobyns AL. Analysis of simply-supported orthotropic plates subject to static and dynamic loads. AIAA J 1998;19(5):642-50. 